\title{
Copy number variation of ZNF280AY across 21 cattle breeds and its association with the reproductive traits of Holstein and Simmental bulls
}

\author{
S. W. Pei, ${ }^{1}$ F. Qin, ${ }^{2}$ W. H. Li ${ }^{1}$ F. D. Li, ${ }^{1,3}$ and X. P. Yue ${ }^{1 *}$ \\ ${ }^{1}$ State Key Laboratory of Grassland Agro-Ecosystems; Key Laboratory of Grassland Livestock Industry Innovation, \\ Ministry of Agriculture and Rural Affairs; Engineering Research Center of Grassland Industry, Ministry of Education; \\ College of Pastoral Agriculture Science and Technology, Lanzhou University, Lanzhou, 730020, P. R. China \\ ${ }^{2}$ School of Pharmacy, Lanzhou University, Lanzhou, 730000, P. R. China \\ ${ }^{3}$ Engineering Laboratory of Sheep Breeding and Reproduction Biotechnology in Gansu Province, Minqin 733300, P. R. China
}

\section{ABSTRACT}

The mammalian Y chromosome gene families in the ampliconic region are expressed predominantly or exclusively in the testis, and their copy number variations (CNV) are significantly associated with male reproductive traits, suggesting they have important roles in spermatogenesis and testicular development. ZNF280AY (zinc finger protein 280A, Y-linked) is a member of the zinc finger protein family and has been identified as a bovid-specific Y-chromosome gene. The current study applied a reliable quantitative real-time PCR method to estimate the CNV of ZNF280AY in 715 bulls across 21 cattle breeds and to further investigate the association of the CNV of ZNF280AY with bull reproductive traits and ZNF280AY mRNA expression levels in adult testis. The results revealed that the median copy number of ZNF280AY was 47, and the copy number varied from 11 to 154, showing significant CNV between and within the investigated cattle breeds. In addition, all 715 bulls were classified into Y1, Y2, and Y3 lineage groups based on a rapid genotyping method described previously. Pairwise comparisons indicated that bulls belonging to the Y1 lineage had a significantly lower median copy number (40) than bulls belonging to the Y2 (52) and Y3 lineages (57). Association analysis revealed that the CNV of ZNF280AY was correlated negatively with the percentage of normal sperm and sperm concentration in Holstein bulls, whereas no significant correlation was observed with ejaculation volume, total sperm count, sperm motility, postthaw motility (PTM), and scrotal circumference in Holstein and Simmental bulls. Furthermore, no correlation was observed between $Z N F 280 A Y$ copy number and $Z N$ F280AY mRNA expression levels in the testis. The

Received November 27, 2018.

Accepted April 23, 2019.

*Corresponding author: lexp@lzu.edu.cn current study suggests that the CNV of the ZNF280AY gene family is associated with male reproductive traits and may serve as a valuable marker for early bull fertility selection in Holstein breeding programs.

Key words: ZNF280AY, copy number variation, reproductive trait, $\mathrm{Y}$ chromosome

\section{INTRODUCTION}

Copy number variation $(\mathbf{C N V})$ is an informative class of common genetic variation, revealing larger genetic effects on phenotypic variability, disease susceptibility, and species evolution than SNP (Zhang et al., 2009; Klopocki and Mundlos, 2011). To date, a large number of studies have been conducted to analyze genome-wide CNV in domestic animals, enabling CNV to be used as an important molecular marker for quantitative traits in genome-wide association studies (GWAS; Hou et al., 2012; Duran Aguilar et al., 2017; Gao et al., 2017). Due to its structural complexity and characteristic of harboring highly repetitive sequences, the Y chromosome has only been sequenced in a very limited number of species, which prevents the incorporation of the $\mathrm{Y}$ chromosome in genome-wide CNV studies.

Based on the studies of $\mathrm{Y}$ chromosome sequencing of human, mouse, and cattle (Skaletsky et al., 2003; Soh et al., 2014; Chang et al., 2013), we found that multicopy gene families are especially prevalent in the male-specific region (MSY) of the mammalian Y chromosome. These multicopy genes show CNV among different individuals. In addition, according to the expression and function analyses, multicopy genes on the mammalian Y chromosome are usually expressed predominantly or solely in the testis and play important roles in spermatogenesis and male fertility (Yue et al., 2014), and CNV of them are significantly associated with male fertility in humans (Giachini et al., 2009; Shen et al., 2013; Yan et al., 2017) and cattle (Hamilton et al., 2009; Yue et al., 2013, 2014). 
On the bovine MSY, 6 multicopy protein-coding genes have been identified (Chang et al., 2013). Of these, TSPY and $H S F Y$ are conserved across different lineages, whereas PRAMEY, ZNF280AY, and ZNF280B $Y$ are bovine-specific (Chang et al., 2013). A previous CNV study on bovine Y-linked genes revealed that the copy number $(\mathbf{C N})$ of PRAMEY can vary within a range of 2 to 30 copies and is negatively associated with testis size, nonreturn rate, and percentage of normal sperm (PNS) in Holstein sires (Yue et al., 2013). In contrast, TSPY, HSFY, and ZNF280BY are largely amplified on the bovine $\mathrm{Y}$ chromosome (up to 350 copies). Association analyses found that the CNV of $H S F Y$ and $Z N F 280 B Y$ correlated negatively with testis size and were positively associated with sire conception rate (Yue et al., 2014), whereas the TSPY CN positively correlated with bull fertility and negatively correlated with TSPY mRNA expression levels in the testis (Hamilton et al., 2012). Generally, all current reports regarding bovine Y-linked $\mathrm{CNV}$ indicate this characteristic may serve as a valuable marker for early bull fertility selection in cattle breeding programs.

ZNF280AY (also known as SUHW2, suppressor of hairy wing homolog 2), as well as ZNF280BY, originated through the autosome-to- $Y$ transposition of a gene block containing ZNF280B and ZNF280A on bovine chromosome 17 (Yang et al., 2011) and was subsequently amplified to $\sim 79$ copies on the bovine Y chromosome (BTAY) of a Hereford bull whose DNA was used for the bovine $\mathrm{Y}$ chromosome sequencing project (Chang et al., 2013). Beyond knowing that its mRNA is expressed predominantly in the spleen and at low levels in the testis and brain of bulls (Yang et al., 2011), the relationship between ZNF280AY and bull fertility still remains elusive. A previous study illustrated that zinc finger proteins have extraordinary diversity of structure and function in mammalian genomes (Laity et al., 2001). Several zinc finger genes have been found to play important roles in mammalian spermatogenesis and male fertility (Noce et al., 1992; Pieler and Bellefroid, 1994). For instance, inactivation of a zinc finger gene in human, ZNF230, leads to failure in spermatogenesis and azoospermia (Zhang et al., 2001). In addition, the ZNF280BY gene family, the paralog of ZNF280AY, showed sense RNA expression in different cell types of the seminiferous tubules, and antisense RNA specific in spermatids, suggesting a role in spermatogenesis (Yang et al., 2011); additionally, its CNV is significantly associated with bull fertility (Yue et al., 2014). Taken together, the CNV of ZNF280AY may be a valuable marker for the selection of elite sires at an early stage.

To date, no study has been conducted to analyze the CNV of ZNF280AY within and among different cattle breeds and its relationship with bull fertility and mRNA expression levels. The objective of this study is to determine whether bovine ZNF280AY CNV differs among individuals and breeds and to unveil the association of ZNF280AY CNV with bull reproductive traits and mRNA expression. Here, we provide evidence that $Z N F 280 A Y$ varies from 11 to 154 copies among 715 bulls from 21 cattle breeds, and a significant association between CNV and bull fertility is observed $(P<0.05)$, whereas no relationship is present between ZNF280AY CNV and its mRNA expression levels in the testis $(\mathrm{r}=-0.078, P=0.704)$.

\section{MATERIALS AND METHODS}

\section{Sample and Reproductive Trait Collection, DNA, and RNA Preparation}

A total of 715 bulls from 21 cattle breeds were collected in this study, including 7 introduced cattle breeds (Angus, Simmental, Holstein, Limousin, Charolais, Gir, and Nelore) with semen or DNA samples and 14 indigenous cattle breeds with blood or tissue samples (ear or testis) in China (Table 1). These introduced cattle breeds have a relatively distant genetic relationship with 14 Chinese indigenous cattle breeds. Of these, semen samples were collected from Holstein $(\mathrm{n}=264)$ and Simmental $(\mathrm{n}=158)$ bulls from the Tianjin Dairy Cattle Center and Gansu Livestock Breeding Center, respectively, who had at least 10 service records of semen quality evaluation, including ejaculation volume (EV), sperm concentration (SPC), total sperm count (TSC), PNS, sperm motility (SM), and postthaw motility (PTM). In addition, scrotal circumference (SC) was measured at 24 mo for 65 Simmental bulls (Supplemental Table S1; https://doi.org/10.3168/jds .2018-16063). The DNA samples of Nelore and Gir bulls were collected from the Bovine HapMap Project (Gibbs et al., 2009). Additionally, blood samples of 10 Holstein cows were collected for negative controls. Genomic DNA was extracted from blood, testis, and ear tissues using a standard phenol-chloroform method (Sambrook et al., 2001), and genomic DNA from semen was extracted using a high-concentration salt procedure (Gannes and Matthew, 2014). Total RNA was isolated from testis samples of 26 Xianan adult bulls (at $24 \mathrm{mo}$ ) by the TRIzol method (TaKaRa, Otsu, Shiga, Japan) and reverse-transcribed with TransScript OneStep gDNA Removal and cDNA Synthesis SuperMix (TransGen Biotech, Beijing, China) using Anchored Oligo $(\mathrm{dT})_{18}$ Primer (TransGen Biotech) according to the manufacturers' guidelines. The concentrations and quality of DNA or RNA were determined by a 
PEI ET AL.

Table 1. Bull information and the median copy number of ZNF280AY in 21 cattle breeds

\begin{tabular}{llccl}
\hline $\begin{array}{l}\text { Breed } \\
\text { full name) }\end{array}$ & $\begin{array}{l}\text { Short } \\
\text { name }\end{array}$ & $\begin{array}{c}\text { Sample } \\
\text { size }\end{array}$ & $\begin{array}{c}\text { Median copy } \\
\text { number (range) }\end{array}$ & $\begin{array}{l}\text { Y chromosome } \\
\text { lineages }\end{array}$ \\
\hline Angus & AGS & 15 & $40(31-84)$ & Y1 \\
Anxi & AX & 14 & $29(16-50)$ & Y2 \\
Bohai & BH & 5 & $56(39-62)$ & Y3 \\
Gir & GIR & 13 & $76(57-134)$ & Y3 \\
Hainan & HN & 6 & $43(30-69)$ & Y3/Y1 \\
Holstein & HS & 264 & $42(11-154)$ & Y1 \\
Jiaxian & JX & 14 & $51(28-84)$ & Y1/Y2/Y3 \\
Limousin & LM & 17 & $55(32-87)$ & Y2 \\
Luxi & LX & 19 & $54(13-77)$ & Y3 \\
Leizhou & LZ & 14 & $63(38-100)$ & Y2/Y3 \\
Mongolian & MG & 17 & $59(45-78)$ & Y2 \\
Nelore & NEL & 5 & $59(46-79)$ & Y3 \\
Nanyang & NY & 29 & $54(36-113)$ & Y2 \\
Qinchuan & QC & 11 & $49(35-69)$ & Y3 \\
Wannan & WN & 7 & $48(35-69)$ & Y3 \\
Xuanhan & XH & 10 & $49(34-90)$ & Y2 \\
Charolais & XL & 34 & $57(38-79)$ & Y2 \\
Simmental & XM & 158 & $53(13-146)$ & Y2 \\
Xianan & XN & 26 & $39(19-57)$ & \\
Tibetan cattle & TC & 19 & $30(15-42)$ & \\
Zaosheng & ZS & 18 & $44(33-89)$ & Th \\
Total & & 715 & $47(11-154)$ & \\
\hline
\end{tabular}

${ }^{1} \mathrm{~A}$ Holstein bull was selected randomly as the calibrator to minimize the interassay variation.

NanoDrop 2000 (Thermo Fisher Scientific, Waltham, MA) and agarose gel electrophoresis and then stored at $-80^{\circ} \mathrm{C}$ for subsequent analysis.

\section{Primer Design}

Based on the annotation of the bovine $\mathrm{Y}$ chromosome sequence, a total of 79 loci for ZNF280AY were identified on the MSY of a Hereford bull (Chang et al., 2013). To retrieve paralogous sequences of all loci, the ZNF280AY sequence (GenBank acc. no. HQ014564) was aligned to the bovine $\mathrm{Y}$ chromosome draft sequence assembly (GenBank acc. no. CM001061.2) using Splign (Kapustin et al., 2008). The retrieved sequences were aligned subsequently to obtain conserved regions for primer design using MEGA 5.0 (Tamura et al., 2011). The primers were designed from conserved regions by the Primer Premier 5.0 program (http:// www.premierbiosoft.com/). The binding sites of the designed primers for ZNF280AY on the draft BTAY assembly (GenBank acc. no. CM001061.2) and the predicted sizes of each amplicon based on In-Silico PCR (http://genome.ucsc.edu/cgi-bin/hgPcr) are listed in Supplemental Table S2 (https://doi.org/10.3168/jds .2018-16063). A single-copy gene, $S R Y$ (sex-determining region $\mathrm{Y}$ ), was used as a reference, and $S R Y$ primers were described in a previous study (Hamilton et al., 2009). The primer information and primer efficiencies can be found in Table 2 .

To validate the male specific amplification of the primers described above, routine PCR was performed to amplify both male and female cattle genomic DNA, and distilled water was used as a blank control. The PCR system contained $12.5 \mu \mathrm{L}$ of $2 \times$ Easy Taq PCR Super MIX (TransGen Biotech), $0.5 \mu \mathrm{L}$ of each primer at $10 \mu \mathrm{mol} / \mu \mathrm{L}, 1 \mu \mathrm{L}$ of $50 \mathrm{ng} / \mu \mathrm{L}$ genomic DNA or

Table 2. Information on the primers used in this study

\begin{tabular}{|c|c|c|c|c|}
\hline Gene & Primer sequence $^{1}\left(5^{\prime}-3^{\prime}\right)$ & $\begin{array}{l}\text { PCR product } \\
\text { size (bp) }\end{array}$ & $\begin{array}{c}\text { Annealing } \\
\text { temperature }\left({ }^{\circ} \mathrm{C}\right)\end{array}$ & $\begin{array}{l}\text { Primer } \\
\text { efficiency }\end{array}$ \\
\hline ZNF280AY & $\begin{array}{l}\text { F: GGAACAGGGTGGGAAACT } \\
\text { R: CTATGGACAGCATACAAGAGAT }\end{array}$ & 203 & 62.5 & 2.00 \\
\hline$G A P D H$ & $\begin{array}{l}\text { F: AATGAAAGGGCCATCACCATC } \\
\text { R: GTGGTTCACGCCCATCACA }\end{array}$ & 204 & 62.5 & - \\
\hline
\end{tabular}

${ }^{1} \mathrm{~F}=$ forward; $\mathrm{R}=$ reverse. 
distilled water, and $10.5 \mu \mathrm{L}$ of distilled water, accounting for a total volume of $25 \mu \mathrm{L}$. Amplification reactions consisted of predenaturation at $95^{\circ} \mathrm{C}$ for $5 \mathrm{~min}$, followed by 35 cycles of denaturation at $94^{\circ} \mathrm{C}$ for $30 \mathrm{~s}$, annealing at $62.5^{\circ} \mathrm{C}$ for $30 \mathrm{~s}$, extension at $72^{\circ} \mathrm{C}$ for $30 \mathrm{~s}$, and a final extension at $72^{\circ} \mathrm{C}$ for $5 \mathrm{~min}$. The PCR products of $S R Y$ and ZNF280AY were separated by gel electrophoresis and visualized under UV illumination (Ge1Doc-It TS Imaging System, Upland, CA).

\section{Determination of Paternal Lineages for Tested Bulls}

Previously, 2 Bos taurus Y haplogroups, Y1 and Y2, and 1 Bos indicus $\mathrm{Y}$ haplogroup (Y3) were identified based on Y-SNP and Y-indels on the X-degenerate region of BTAY (Edwards et al., 2011). Afterward, Bonfiglio et al. developed a rapid and unambiguous method to classify the above 3 bovine Y-haplogroups using 2 polymorphisms within USP9Y (ubiquitin specific peptidase 9, Y-linked; Bonfiglio et al., 2012). Briefly, an 81-bp insertion can first distinguish the Y1 haplogroup from the Y2 and Y3 haplogroups by PCR and electrophoresis. Subsequently, Y3 can be differentiated from Y2 with an $S s p$ I endonuclease digestion of the same PCR product containing a Y-SNP (g.76426C > T; Supplemental Figure S1; https://doi.org/10.3168/ jds.2018-16063). The detailed procedure to determine the $3 \mathrm{Y}$ lineages for all bulls in this study followed the previous description (Bonfiglio et al., 2012).

\section{CNV Estimation and mRNA Expression Levels of ZNF280AY}

Quantitative real-time PCR (qPCR) was used to measure the CN of ZNF280AY for each bull using a CFX96 real-time PCR detection system (Bio-Rad, Hercules, CA) and SYBR Premix Ex TaqII Kit (TaKaRa, Dalian, China). Standard curves were applied to evaluate primer efficiencies from a series of the DNA dilution of 40, 20, 10, 5 , and $2.5 \mathrm{ng} / \mu \mathrm{L}$ for both $Z N F 280 A Y$ and $S R Y$ primers. The resulting reactions had efficiencies of 2.00 and 1.95 for $Z N F 280 A Y$ and $S R Y$, respectively, according to the equation $\mathrm{E}=10^{-1 / \text { slope }}$ (Hamilton et al., 2009; Yue et al., 2013). For both genes, a 10- $\mu \mathrm{L}$ reaction system, containing $1 \mu \mathrm{L}$ of DNA $(5 \mathrm{ng} / \mu \mathrm{L}), 5 \mu \mathrm{L}$ of SYBR Premix Ex TaqII (TaKaRa), $0.5 \mu \mathrm{L}$ of each primer at $10 \mu \mathrm{mol} / \mu \mathrm{L}$, and $3 \mu \mathrm{L}$ of distilled $\mathrm{H}_{2} \mathrm{O}$ was run with the following PCR program: predenaturation at $95^{\circ} \mathrm{C}$ for $10 \mathrm{~min}$, followed by 45 cycles of denaturation at $95^{\circ} \mathrm{C}$ for $15 \mathrm{~s}$, annealing at $62.5^{\circ} \mathrm{C}$ for $30 \mathrm{~s}$, and extension at $72^{\circ} \mathrm{C}$ for $30 \mathrm{~s}$, with a final extension at $72^{\circ} \mathrm{C}$ for $5 \mathrm{~min}$. A melting curve was then generated by taking fluorescent measurements every $0.1^{\circ} \mathrm{C}$ from 60 to $95^{\circ} \mathrm{C}$. Within each 96-well PCR plate, one fixed bull sample was set as a calibrator to adjust the interassay variation. In addition, one female genomic DNA sample and one distilled water sample were used as a negative and a blank control, respectively. The CN of ZNF280AY test samples was estimated with 3 equations described previously (Hamilton et al., 2009; Yue et al., 2013).

The ZNF280AY mRNA expression levels in the testes of 26 Xianan bulls were estimated using the $2^{-\Delta \Delta \mathrm{Ct}}$ method (Schmittgen and Livak, 2008) with GAPDH as an internal standard. The primers used for mRNA expression were described in a previous study (Yang et al., 2011). The $\mathrm{qPCR}$ reaction was run with the same components as those used for CNV estimation, with cDNA (20 ng/ $\mu \mathrm{L})$ replacing DNA, under the following program: predenaturation at $95^{\circ} \mathrm{C}$ for $3 \mathrm{~min}$, followed by 40 cycles of denaturation at $95^{\circ} \mathrm{C}$ for $10 \mathrm{~s}$, annealing at $62.5^{\circ} \mathrm{C}$ for $30 \mathrm{~s}$, extension at $72^{\circ} \mathrm{C}$ for $30 \mathrm{~s}$, and a final extension at $72^{\circ} \mathrm{C}$ for $5 \mathrm{~min}$. The qPCR for each sample was run in triplicate.

\section{Association and Statistical Analysis}

To minimize technical error and to obtain accurate CN estimates, test samples were removed from further analysis if the raw qPCR data showed a coefficient of variation $(\mathbf{C V})>1 \%$ between triplicates. The normal distribution of the ZNF280AY CN data was tested with the Kolmogorov-Smirnov and Shapiro-Wilk normality tests (Shapiro and Wilk, 1965; Justel et al., 1997). Conventional box plot analyses were implemented with the $\mathrm{CN}$ data to detect the outliers. Multiple pairwise comparison of the ZNF280AY median copy number (MCN) between breeds was performed using a nonparametric Mann-Whitney U test (Mann and Whitney, 1947), with a Bonferroni correction for multiple testing (Dunn, 1961). A 2-factor nested ANOVA model was used to compare the MCN among groups that were classified based on the Y1, Y2, and Y3 lineages of cattle individuals, with the breed nesting within $\mathrm{Y}$ lineage group, using the SAS 9.2 software (SAS Institute Inc., Cary, NC).

Association analysis between ZNF280AY CNV and reproductive traits was performed for Holstein and Simmental bulls using the Pearson correlation test in SPSS 17.0 (SPSS Inc., Chicago, IL). Furthermore, a general linear model in SAS 9.2 was applied to investigate the effect of CNV of ZNF280AY and breed, and their interaction on EV, SPC, and TSC. The Student $t$-test was conducted to compare reproductive traits between 20 Holstein bulls with the highest $\mathrm{CN}$ and 20 Holstein bulls with lowest CN of ZNF280AY. Additionally, the correlation between ZNF280AY CNV and its mRNA expression levels was analyzed using the Spearman test 


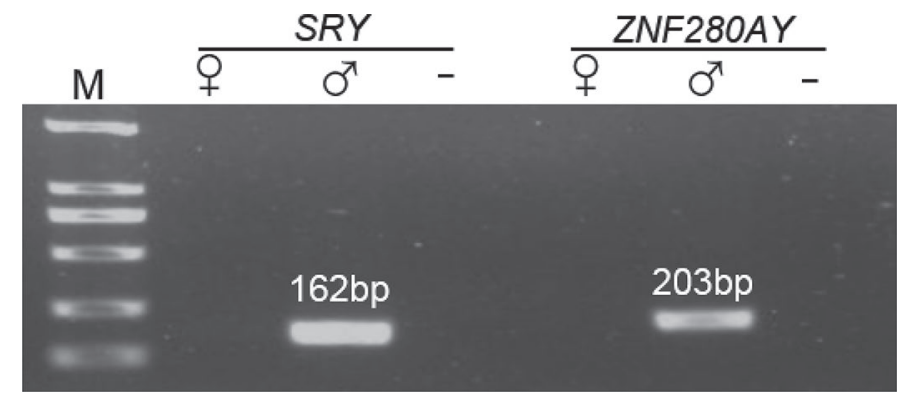

Figure 1. Gel electrophoresis of PCR products of the bovine $Z N F 280 A Y$ and $S R Y$ genes. The primers of these $2 \mathrm{Y}$-linked genes amplified male-specific bands; expected fragment size is labeled above each band. $\mathrm{M}=2$-kb DNA ladder; $\sigma^{\top}=$ male cattle genomic DNA; 우 $=$ female cattle genomic DNA; - = negative control (distilled water).

of SPSS 17.0. A $P$-value of $<0.05$ was considered statistically significant for all tests except for the multiple pairwise comparison of the ZNF280AY MCN between breeds.

\section{RESULTS}

\section{CNV of ZNF280AY Across Cattle Breeds}

Before estimating ZNF280AY CN and mRNA expression levels, the male specificity of the ZNF280AY and $S R Y$ primers was validated by routine PCR. The results revealed that primers for both genes showed amplification only in male genomic DNA with expected fragment sizes, confirming that the designed primers are male specific (Figure 1) and can be used for $Z N$ F280AY CNV estimation.

Based on the equations for CNV calculation described previously (Hamilton et al., 2009; Yue et al., 2013), the $\mathrm{CN}$ of ZNF280AY was 41 for the calibrator sample. Subsequently, significant CNV was observed between and within the investigated cattle breeds. The MCN of 715 bulls from 21 cattle breeds was 47, ranging from 11 to 154 copies (Table 1). According to the KolmogorovSmirnov and Shapiro-Wilk normality tests, the CN data for all cattle breeds did not fit a normal distribution $(P<0.05)$. Both Holstein and Simmental had large sample sizes, and the total populations of the 21 cattle breeds showed several outliers based on box plot analyses (Figure 2).

Pairwise comparisons were applied to compare MCN between the tested cattle breeds, which revealed significant differences in MCN between some cattle breeds (Supplemental Table S3; https://doi.org/10.3168/jds .2018-16063). Of those, Gir bulls possessed the highest MCN (76 copies, range: 57-134), whereas Anxi bulls showed the lowest MCN (29 copies, range: 16-50; Table 1). It was interesting to note that the $\mathrm{CN}$ of $Z N F 280 A Y$ in Tibetan bulls (30 copies, range: $15-42$ ) was significantly different $(P<0.001)$ from that of most other breeds (Supplemental Table S3; https://doi.org/10 .3168/jds.2018-16063).

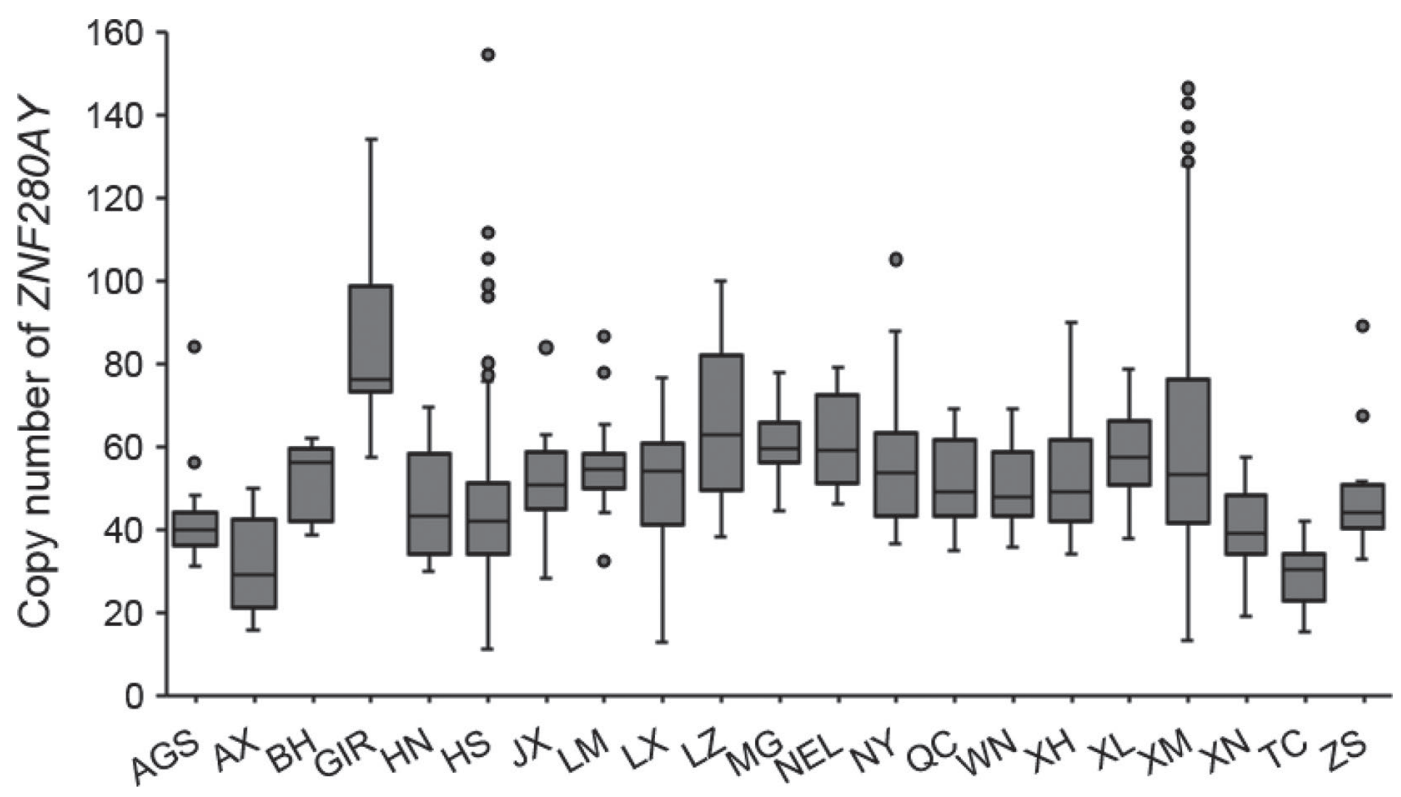

Figure 2. Box plot analysis of copy number of ZNF280AY in cattle breeds. The median copy number of ZNF280AY is indicated by a central line in a box, and the black dots represent outlines. The top and bottom of a box and whiskers indicate the first and third quartiles (the 25th and 75th percentiles). The x-axis represents the different cattle breeds. AGS = Angus; AX = Anxi; BH = Bohai; GIR = Gir; HN = Hainan; $\mathrm{HS}=$ Holstein; JX = Jiaxian; LM = Limousin; LX = Luxi; LZ = Leizhou; MG = Mongolian; NEL = Nelore; NY = Nanyang; QC = Qinchuan; $\mathrm{WN}=$ Wannan; $\mathrm{XH}=$ Xuanhan; $\mathrm{XL}=$ Charolais; $\mathrm{XM}=$ Simmental; XN = Xianan; TC $=$ Tibetan cattle; ZS $=$ Zaosheng. 


\section{Comparison of CN Among Cattle Breeds with Different Y Lineages}

The 21 cattle breeds in current study can be classified into 4 groups based on genotyping results using 2 polymorphisms in USP9Y, including the Y1-lingeage group (Angus, Holstein, and Tibetan cattle), Y2-lineage group (Anxi, Limousin, Mongolian, Qinchuan, Charolais, Simmental, Xianan, and Zaosheng), Y3-lineage group (Bohai, Gir, Luxi, Nelore, Wannan, and Xuanhan), and combined Y-lineages group (Hainan, Jiaxian, Leizhou, and Nanyang; Table 1, Supplemental Table S4; https:// doi.org/10.3168/jds.2018-16063). To explore the potential relationship between the CNV of ZNF280AY and paternal lineages, the MCN was compared among the 3 different $\mathrm{Y}$ lineages. Bulls with the $\mathrm{Y} 1$ lineage showed a significantly lower MCN (40 copies, range of 11 to 154) than bulls with the Y2 lineage (52 copies, range of 13 to 146) and Y3 lineage (57 copies, range of 13 to 134; $P<0.001)$. No significant difference was observed between the Y2 and Y3 lineages $(P>0.05)$, which was further confirmed in the combined Y-lineages group of the Nanyang cattle breed. Among 29 Nanyang cattle, 18 individuals with Y2 possessed a MCN of ZNF280AY similar to that of 11 individuals with Y3 (Figure 3). In addition, the low MCN of ZNF280AY in the Y1 lineage revealed that Bos taurus (BTA) bulls had a significantly lower MCN (46 copies, range of 11 to 154) than that of Bos indicus (BIN) bulls (57 copies, range of 13 to $134 ; P<0.001$ ), potentially because $\mathrm{Y} 1$ and $\mathrm{Y} 2$ are BTA lineages and Y3 is a BIN lineage (Figure 3).

\section{Association Analysis Between ZNF280AY CNV and Bull Reproductive Traits}

To obtain robust associations between the $\mathrm{CNV}$ of ZNF280AY and bull reproductive traits, Holstein and Simmental bulls with at least 10 service records from different seasons were included in association analyses. In 142 Holstein bulls, the CNV of ZNF280AY was significantly correlated with PNS $(\mathrm{r}=-0.179, P=0.016$; Table 3, Figure 4A), whereas no significant correlation was found between the CNV of ZNF280AY and the remaining semen quality parameters, including SPC, EV, TSC, SM, and PTM $(P>0.05$; Table 3). In 107 Simmental bulls, the CNV of ZNF280AY had no significant relationship with either semen quality parameters or SC $(P>0.05$; Table 3$)$. In addition, 4 outliers were found among the above 142 Holstein bulls based on box plot analyses. To investigate whether the significant association was driven by outliers, these 4 outliers were excluded from a re-association analysis of the remaining 138 Holstein bulls. Notably, stronger correlations were found between the CNV of ZNF280AY and SPC ( $\mathrm{r}=$
$-0.148, P=0.042)$ and PNS $(\mathrm{r}=-0.206, P=0.008$; Table 3, Figure 4B, C), indicating that the low CN of ZNF280AY was beneficial for Holstein bull fertility. For Simmental bulls, no significant association with SC measurement was identified after removing 3 outliers from 65 bulls $(P>0.05$; Table 3$)$.

To further investigate the effect of low and high CN of ZNF280AY on the bull productive traits, semen quality of Holstein bulls was compared between 20 bulls with the highest $\mathrm{CN}$ (high CN group; $\mathrm{CN} \geq 55$ ) and 20 bulls with the lowest $\mathrm{CN}$ (low $\mathrm{CN}$ group; $\mathrm{CN} \leq 30$ ) of ZNF280AY by a $t$-test. No difference was observed in EV, SPC, TSC, SM, and PTM for Holstein bulls between the high and low $\mathrm{CN}$ groups $(P>0.05)$, but PNS for the low CN group was significantly higher than for the high CN group $(P=0.016$; Table 4$)$, which was in accordance with Pearson correlation results.

\section{Relationship Between ZNF280AY CN and mRNA Expression}

The mRNA expression levels of ZNF280AY were evaluated in the testes of 26 Xianan adult bulls using the $2^{-\Delta \Delta \mathrm{Ct}}$ method and then correlated with the $\mathrm{CN}$ of ZNF280AY in corresponding Xianan bulls. The results revealed no significant relationship between $Z N F 280 A Y$ $\mathrm{CN}$ and mRNA expression levels $(\mathrm{r}=-0.078, P=$ $0.704)$, suggesting no dosage effect in the testis.

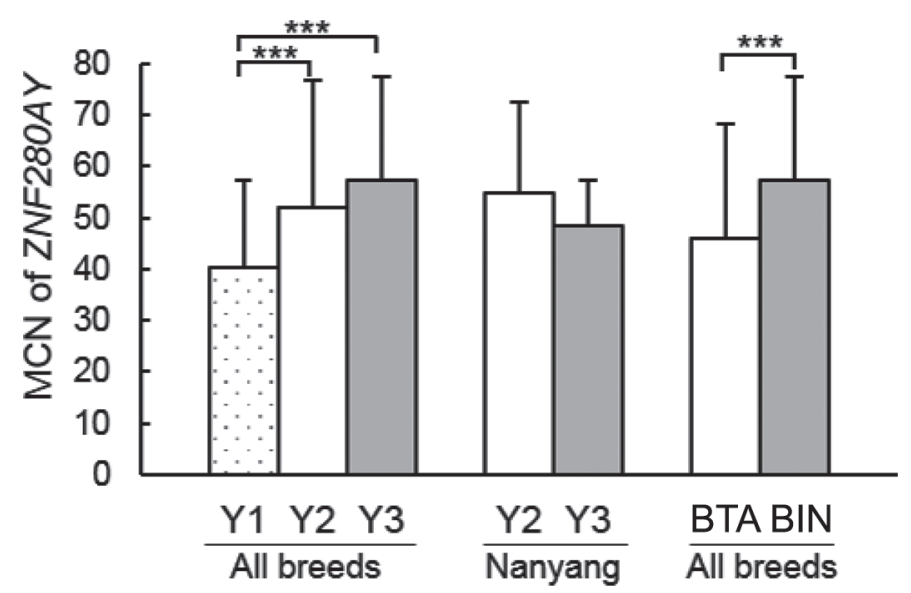

Figure 3. Comparison of the median copy number (MCN) of ZNF280AY between bulls with 3 major Y chromosome lineages (Y1, Y2, and Y3) or 2 paternal lineages [Bos taurus (BTA) and Box indicus (BIN)]. The MCN of ZNF280AY was significantly different between the $\mathrm{Y}$ chromosome lineages or paternal lineages $(P<0.001)$. The MCN of the Y3 lineage was significantly higher than those of the Y1 and Y2 lineages $(P<0.001)$, whereas MCN was not significantly different between the Y2 and Y3 lineages. Similarly, no significant differences were found between the Y1 and Y2 lineages in the Nanyang cattle $(P>0.05)$. The BTA-derived bulls had a significantly lower MCN of ZNF280AY than BIN-derived bulls $(P<0.001)$. The error bars represent SE. ${ }^{* * *} P<0.001$. 


\section{DISCUSSION}

In this study, a robust qPCR method was applied to evaluate the CNV of ZNF280AY across 21 cattle breeds. This method has been shown to provide a very effective and accurate measurement of CNV because it takes full advantage of the public bovine $\mathrm{Y}$ chromosome assembly (CM001061.2), which enables us to design primers on the conserved regions of different ZNF280AY loci (Yue et al., 2014). In previous studies, the CN of $H S F Y$ was first estimated to be 74 copies in Holstein bulls without CNV (Hamilton et al., 2011), whereas a later study found that the CN of HSFY varied from 21 to 308 using the same method as the current study (Yue et al., 2014). In addition, the $\mathrm{CN}$ of 3 bovine Y-linked genes, PRAMEY, HSFY, and ZNF280BY, were previously determined to be 10,192 , and 234, respectively, in the bull L1 Domino 99375, which was used for bovine Y chromosome sequencing (Chang et al., 2013), and the current qPCR method estimated the $\mathrm{CN}$ for this particular bull to be 13, 204, and 239, respectively (Yue et al., 2013, 2014). Considering some unavoidable experimental errors, these CNV estimations are very reliable. Using this reliable qPCR method, the current study first found that $Z N F 280 A Y$ showed a wide range of $\mathrm{CNV}$ in 21 cattle breeds, and significant differences were observed in CN between and within cattle breeds (Table 1; Supplemental Table S3, https://doi.org/10 $.3168 /$ jds.2018-16063).

According to the differences in the MCN of $Z N$ F280AY among cattle breeds, we found that the MCN of Tibetan bulls was estimated at 30 copies, which was significantly lower than the MCN of other cattle breeds, except Anxi (29 copies; Table 1), and remarkably divergent from those of other cattle breeds (Supplemental Table S3; https://doi.org/10.3168/jds.2018-16063).
This finding may be due to differences in evolutionary selection pressure during the domestication process between different cattle breeds. Tibetan bulls have a restricted distribution in the Tibet Autonomous Region of China and have strong adaptive abilities for highaltitude environments (Song et al., 2016), which have led to the accumulation of a large number of domestication signals during long-term natural selection. Copy number variation is a predominant source of genomic structural variation and plays an important role in evolutionary diversification and domestication (Zhang et al., 2009, 2015). We anticipate that our findings in part reflect that the ZNF280AY gene evolved under stronger purifying selection in Tibetan bulls (Zhang et al., 2009), which could be interpreted as a signature of adaptation to different local or climatic conditions (Letaief et al., 2017). Simultaneously, our findings also provide new insight into the contribution of Y-linked multicopy genes to evolutionary selection pressure beyond reproductive traits (Hughes and Page, 2015).

Similar to a previous report, CNV were confirmed to be associated with species evolution, and some CNV are lineage specific ( $\mathrm{Li}$ et al., 2013). In the present study, we found that the CN patterns of ZNF280AY vary greatly in different paternal lineages among the 21 breeds tested. The MCN of ZNF280AY was significantly reduced $(P<0.001)$ in the Y1-lineage group $(40$ copies) relative to those in the Y2-lineage (52 copies) and Y3-lineage groups (57 copies), and further analysis showed that the CN of ZNF280AY was highly expanded in the BIN lineage and reduced in the BTA lineage (57 copies vs. 46 copies; $P<0.001 ;$ Figure 3 ). This finding is congruent with previous reports indicating that the CN of multicopy gene families is significantly different between the BTA and BIN lineages (Chang et al., 2013). For instance, the MCN of ZNF280BY and

Table 3. Pearson correlation between the copy number of ZNF280AY and reproductive traits in the Holstein and Simmental populations

\begin{tabular}{|c|c|c|c|c|c|}
\hline \multirow[b]{2}{*}{ Breed } & \multirow{2}{*}{$\begin{array}{l}\text { Reproductive } \\
\text { trait }^{1}\end{array}$} & \multicolumn{2}{|c|}{ With outliers } & \multicolumn{2}{|c|}{ Without outliers $^{2}$} \\
\hline & & $\mathrm{r}$ & $P$-value & $\mathrm{r}$ & $P$-value \\
\hline \multirow[t]{6}{*}{ Holstein } & $\mathrm{EV}$ & -0.066 & 0.216 & -0.026 & 0.382 \\
\hline & $\mathrm{SPC}$ & -0.081 & 0.169 & -0.148 & 0.042 \\
\hline & TSC & -0.081 & 0.169 & -0.093 & 0.139 \\
\hline & SM & -0.007 & 0.467 & -0.040 & 0.321 \\
\hline & PNS & -0.179 & 0.016 & -0.206 & 0.008 \\
\hline & PTM & 0.008 & 0.460 & 0.004 & 0.482 \\
\hline \multirow[t]{4}{*}{ Simmental } & $\mathrm{EV}$ & -0.090 & 0.286 & -0.090 & 0.286 \\
\hline & $\mathrm{SPC}$ & -0.101 & 0.261 & -0.101 & 0.261 \\
\hline & TSC & -0.042 & 0.397 & -0.042 & 0.397 \\
\hline & $\mathrm{SC}$ & 0.089 & 0.239 & 0.142 & 0.135 \\
\hline
\end{tabular}

${ }^{1} \mathrm{EV}=$ ejaculation volume; $\mathrm{SPC}=$ sperm concentration; $\mathrm{TSC}=$ total sperm count; $\mathrm{SM}=$ sperm motility; PNS $=$ percentage of normal sperm; PTM = postthaw motility; $\mathrm{SC}=$ scrotal circumference.

${ }^{2}$ Four and 3 outlier bulls identified in the box plot analysis were excluded from the correlation analysis in the Holstein and Simmental populations, respectively. 


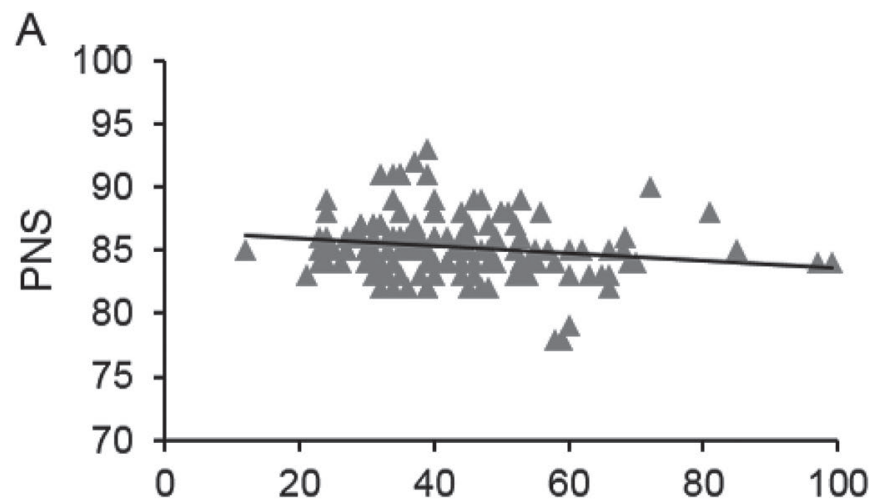

B

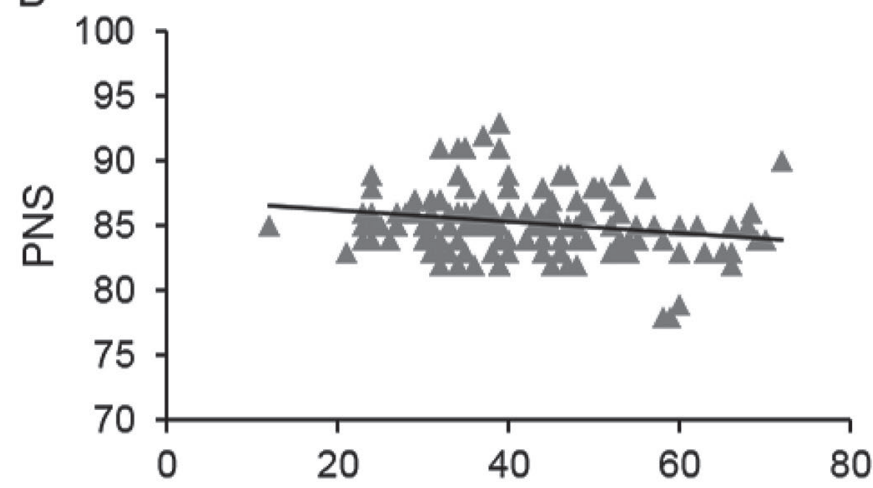

C

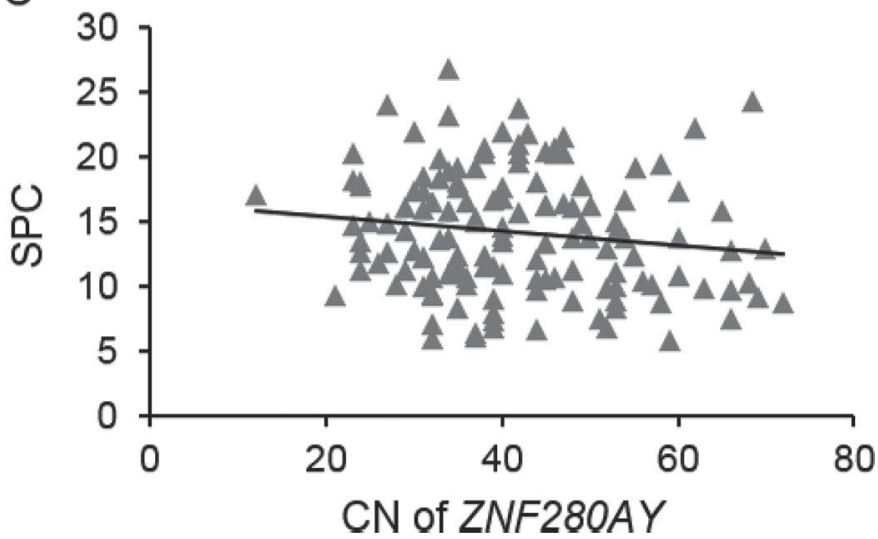

Figure 4. Scatter diagram reflecting the correlations between copy number $(\mathrm{CN})$ variation $(\mathrm{CNV})$ of $Z N F 280 A Y$ and male reproductive traits. The CNV of ZNF280AY was negatively associated with (A) percentage of normal sperm (PNS; $\mathrm{r}=-0.179, P=0.016$ ), (B) PNS (r $=-0.206, P=0.008$; excluding 4 outliers), and (C) sperm concentration (SPC; $\mathrm{r}=-0.148, P=0.042$ ). Each gray triangle represents one individual.

PRAMEY in the BTA lineage were significantly higher than those in the BIN lineage (Yue et al., 2013, 2014). In contrast, the MCN of HSFY in the BTA lineage was significantly lower than that in the BIN lineage (Yue et al., 2013). These results lead us to believe that the $\mathrm{CN}$ of multicopy genes are associated with paternal lineages in cattle. Therefore, it is necessary to consider the effects of paternal lineage when investigating the CNV of multicopy gene families among the $\mathrm{Y}$ lineages (Yan et al., 2017).

However, the mechanism underlying the differences in $\mathrm{CN}$ for gene families in different $\mathrm{Y}$ lineages is still unclear. As a consequence of the distinct evolution of the bovine $\mathrm{Y}$ chromosome, the morphology of the $\mathrm{Y}$ chromosome between B. taurus and B. indicus is remarkably different, as corroborated by fluorescence in situ hybridization, which revealed that the BAT Y chromosome is submetacentric, whereas the BIN Y chromosome is acrocentric (Goldammer et al., 1997). This remarkable difference is possibly due to a series of cytological events, including centromere transposition, chromosome segment transpositions, gain or loss of constitutive heterochromatin, and pericentric inversions (Iannuzzi et al., 2009). This morphological difference in different lineages likely will lead to diversified gene content. If so, we suggest that the differences in CN among Y chromosome gene families between the BTA and BIN lineages has accelerated the structural remodeling of Y chromosome morphology.

Recently, the mechanism of intrapalindrome gene conversion was theoretically verified, and this mechanism can generate $\mathrm{CN}$ changes in the human Y chromosome (Trombetta and Cruciani, 2017; Shi et al., 2018). However, the driving mechanism behind the large expansion and CNV of multicopy genes on the bovine $\mathrm{Y}$ chromosome remains unknown. Previous studies have suggested that the unique features identified in the primate MSY are palindromes, based on Y chromosome sequencing, and provide a basis for $\mathrm{Y}$-to- $\mathrm{Y}$ gene conversion (Chang et al., 2013). The transcriptional landscape of the bovine MSY has revealed that approximately 80 palindrome-like repeat units $(\sim 420 \mathrm{~kb}$ per unit) constitute the bovine $\mathrm{Y}$ ampliconic region, and each unit resides in 4 multicopy families (TSPY, $Z N$ F280AY, ZNF280BY, and HSFY; Chang et al., 2013), which provides an important structural foundation in favor of intra- and interpalindrome gene conversion and potentially regulates the equilibrium between gene loss and acquisition during Y chromosome evolution (Trombetta and Cruciani, 2017). Notably, regardless of the underlying cause of the temporal disparity in multicopy gene families (Oluwole et al., 2017), when combined with the significant differences in CNV of ZNF280AY across cattle breeds, we believe that the highly variable $\mathrm{CN}$ of this gene is most likely due to frequent gene conversion within the $\sim 80$ palindrome-like region.

Currently, the Y chromosome research hotspot mainly focuses on possible associations between male fertility and CNV in multicopy, testis-specific gene families (Hughes and Page, 2015). The majority of studies on 
the association between the CNV of $\mathrm{Y}$ chromosome testis-specific gene families and male fertility have been proposed to look for some valuable molecular markers for male fertility selection or the diagnosis of male reproductive disorders. The 2 previous studies showed that the CNV of HSFY, ZNF280BY, and PRAMEY are negatively correlated with testis size (SC, age-adjusted relative SC) in Holstein bulls (Yue et al., 2013, 2014). A recent study on the mechanism of cattle-yak hybrid bull infertility indicated that the tremendous expansion of TSPY CN in hybrid bulls significantly influenced the success of spermatogenesis, which may be a potential risk factor for hybrid infertility (Zhang et al., 2016).

Our study revealed that the CNV of ZNF280AY was negatively associated with SPC and PNS in the Holstein population, suggesting that a bull with a higher CN of ZNF280AY tends to have worse semen quality. In contrast, a trend toward a negative correlation between ZNF280AY CN and SPC $(\mathrm{r}=-0.101, P=$ 0.261; Table 3) was found in the Simmental population, even though CNV was not significantly associated with semen quality or testicular size $(P>0.05)$. This result might be explained by the fact that the sample size of the Simmental population used for correlation analysis was relatively small and included limited phenotypic data for the assessment of reproductive performance. In addition, differences in genetic background between 2 cattle breeds can also cause bias. Therefore, it would be interesting to study the correlation between CNV and male fertility by performing large-scale phenotyping and assessing diverse types of male reproductive traits.

Gene dosage is the major molecular mechanism that conveys phenotypes associated with CNV (Zhang et al., 2009; Zhou et al., 2011). In most cases, CNV can alter the expression levels of functional genes and affect consequent phenotypes; such genes are described as dosage sensitive. For instance, CNV of spermatogenesis-related genes (RBMY1 and TSPY) were closely associated with their levels of gene transcription and translation, and their duplication led to increased or decreased mRNA and protein levels (Hamilton et al., 2012; Yan et al., 2017). In this study, the CNV of ZNF280AY did not show a relationship with its mRNA expression levels in the testis, indicating that ZNF280AY may have not a dosage effect in the testis, and in fact, previous research showed that the spleen is the tissue with the highest ZNF280AY expression (Yang et al., 2011). Therefore, this correlation should be done in the spleen to check whether a dosage effect exists in a future study. In addition, a previous analysis of ZNF280AY genomic sequences predicted a short or no open reading frame. It has been speculated that the majority of the ZNF280AY loci on BTA Y chromosomes were pseudogenized over longer time periods (Yang et al., 2011). High sequence similarity (95-100\%) between functional copies of $Z N F 280 A Y$ and the large number of pseudogenes potentially bias our CN detection (Yan et al., 2017). Until recently, there was no effective way to distinguish functional copies from pseudogenes. Therefore, it is challenging to design primers that capture only functional copies of the ZNF280AY gene as well as determine the relationship between $Z N F 280 A Y \mathrm{CN}$ and gene expression levels. Furthermore, comprehensive understandings of gene function are ultimately required to detect functional copies and determine the correlation between ZNF280AY CN and its mRNA/protein levels in testis tissue as well as the subcellular location of the ZNF280AY protein.

\section{CONCLUSIONS}

The present study is the first to provide evidence that ZNF280AY was extensively expanded and showed CNV between and within different cattle breeds. An association study found that the CNV of ZNF280AY was negatively associated with sperm quality as measured by PNS and SPC, implying that ZNF280AY may play an important role in sperm maturation and providing valuable insight into early selection for bull fertility of Holstein dairy cattle.

Table 4. Comparison of reproductive traits between Holstein bulls with the high and low copy numbers $(\mathrm{CN}$; mean $\pm \mathrm{SE}$ ) of $Z N F 280 A Y^{1}$

\begin{tabular}{lccc}
\hline Productive trait $^{2}$ & $\begin{array}{c}\text { Low } \mathrm{CN} \\
(\mathrm{CN} \leq 30, \mathrm{n}=20)\end{array}$ & $\begin{array}{c}\text { High CN } \\
(\mathrm{CN} \geq 55, \mathrm{n}=20)\end{array}$ & $P$-value \\
\hline EV & $6.78 \pm 0.54$ & $6.69 \pm 0.58$ & 0.904 \\
SPC & $14.78 \pm 0.82$ & $12.93 \pm 1.15$ & 0.197 \\
TSC & $102.10 \pm 10.75$ & $90.18 \pm 13.50$ & 0.494 \\
PNS & $85.55 \pm 0.32$ & $83.70 \pm 0.66$ & 0.016 \\
SM & $82.60 \pm 1.66$ & $81.00 \pm 1.79$ & 0.517 \\
PTM & $41.20 \pm 0.29$ & $41.50 \pm 0.30$ & 0.477 \\
\hline
\end{tabular}

${ }^{1}$ The comparison was conducted by Student's $t$-tests.

${ }^{2} \mathrm{EV}=$ ejaculation volume; $\mathrm{SPC}=$ sperm concentration; $\mathrm{TSC}=$ total sperm count; $\mathrm{PNS}=$ percentage of normal sperm; $\mathrm{SM}=$ sperm motility; PTM = postthaw motility. 


\section{ACKNOWLEDGMENTS}

This work was supported by National Natural Science Foundation of China (31501918) and the Program for Changjiang Scholars and Innovative Research Team in University (IRT13019). We are grateful to Wansheng Liu at Pennsylvania State University for sharing DNA samples of Nelore and Gire bulls used in the Bovine HapMap Project.

\section{REFERENCES}

Bonfiglio, S., A. De Gaetano, K. Tesfaye, V. Grugni, O. Semino, and L. Ferretti. 2012. A novel USP9Y polymorphism allowing a rapid and unambiguous classification of Bos taurus $\mathrm{Y}$ chromosomes into haplogroups. Anim. Genet. 43:611-613.

Chang, T., Y. Yang, E. F. Retzel, and W.-S. Liu. 2013. The malespecific region of the bovine $\mathrm{Y}$ chromosome is gene-rich with a high transcriptomic activity in testis development. Proc. Natl. Acad. Sci. USA 110:12373-12378.

Dunn, O. J. 1961. Multiple comparisons among means. Publ. Am. Stat. Assoc. 56:52-64.

Duran Aguilar, M., S. I. Roman Ponce, F. J. Ruiz Lopez, E. Gonzalez Padilla, C. G. Vasquez Pelaez, A. Bagnato, and M. G. Strillacci. 2017. Genome-wide association study for milk somatic cell score in Holstein cattle using copy number variation as markers. J. Anim. Breed. Genet. 134:49-59.

Edwards, C. J., G. Catarina, K. Juha, P. P. Lucía, T. Anne, S. Frauke, L. T. Gama, M. C. T. Penedo, D. G. Bradley, and J. A. Lenstra. 2011. Dual origins of dairy cattle farming-Evidence from a comprehensive survey of European Y-chromosomal variation. PLoS One 6:e15922.

Gannes, D., and K. Matthew. 2014. Rapid method of processing sperm for nucleic acid extraction in clinical research. MS Thesis. Department of Public Health, University of Massachusetts, Amherst.

Gao, Y., J. Jiang, S. Yang, Y. Hou, G. E. Liu, S. Zhang, Q. Zhang, and D. Sun. 2017. CNV discovery for milk composition traits in dairy cattle using whole genome resequencing. BMC Genomics 18:265.

Giachini, C., F. Nuti, D. J. Turner, I. Laface, Y. Xue, F. Daguin, G. Forti, C. Tyler-Smith, and C. Krausz. 2009. TSPY1 copy number variation influences spermatogenesis and shows differences among Y lineages. J. Clin. Endocrinol. Metab. 94:4016-4022.

Gibbs, R. A., J. F. Taylor, C. P. Van Tassell, W. Barendse, K. A. Eversole, C. A. Gill, R. D. Green, D. L. Hamernik, S. M. Kappes, S. Lien, L. K. Matukumalli, J. C. McEwan, L. V. Nazareth, R. D. Schnabel, G. M. Weinstock, D. A. Wheeler, P. Ajmone-Marsan, P. J. Boettcher, A. R. Caetano, J. F. Garcia, O. Hanotte, P. Mariani, L. C. Skow, T. S. Sonstegard, J. L. Williams, B. Diallo, L. Hailemariam, M. L. Martinez, C. A. Morris, L. O. C. Silva, R. J. Spelman, W. Mulatu, K. Zhao, C. A. Abbey, M. Agaba, F. R. Araujo, R. J. Bunch, J. Burton, C. Gorni, H. Olivier, B. E. Harrison, B. Luff, M. A. Machado, J. Mwakaya, G. Plastow, W. Sim, T. Smith, M. B. Thomas, A. Valentini, P. Williams, J. Womack, J. A. Woolliams, Y. Liu, X. Qin, K. C. Worley, C. Gao, H. Jiang, S. S. Moore, Y. Ren, X.-Z. Song, C. D. Bustamante, R. D. Hernandez, D. M. Muzny, S. Patil, A. San Lucas, Q. Fu, M. P. Kent, R. Vega, A. Matukumalli, S. McWilliam, G. Sclep, K. Bryc, J. Choi, H. Gao, J. J. Grefenstette, B. Murdoch, A. Stella, R. Villa-Angulo, M. Wright, J. Aerts, O. Jann, R. Negrini, M. E. Goddard, B. J. Hayes, D. G. Bradley, M. Barbosa da Silva, L. P. L. Lau, G. E. Liu, D. J. Lynn, F. Panzitta, and K. G. Dodds. 2009. Genomewide survey of SNP variation uncovers the genetic structure of cattle breeds. Science 324:528-532.

Goldammer, T., R. M. Brunner, and M. Schwerin. 1997. Comparative analysis of $\mathrm{Y}$ chromosome structure in Bos taurus and B. indicus by FISH using region-specific, microdissected, and locus-specific DNA probes. Cytogenet. Cell Genet. 77:238-241.
Hamilton, C. K., L. A. Favetta, G. P. Di Meo, S. Floriot, A. Perucatti, J. Peippo, J. Kantanen, A. Eggen, L. Iannuzzi, and W. A. King. 2009. Copy number variation of testis-specific protein, Y-encoded (TSPY) in 14 different breeds of cattle (Bos taurus). Sex Dev. 3:205-213.

Hamilton, C. K., T. Revay, R. Domander, L. A. Favetta, and W. A. King. 2011. A large expansion of the $H S F Y$ gene family in cattle shows dispersion across $\mathrm{Yq}$ and testis-specific expression. PLoS One 6:e17790.

Hamilton, C. K., A. R. Verduzco-Gómez, L. A. Favetta, P. Blondin, and W. A. King. 2012. Testis-specific protein Y-encoded copy number is correlated to its expression and the field fertility of Canadian Holstein bulls. Sex Dev. 6:231-239.

Hou, Y., G. E. Liu, D. M. Bickhart, L. K. Matukumalli, C. Li, J. Song, L. C. Gasbarre, C. P. V. Tassell, and T. S. Sonstegard. 2012. Genomic regions showing copy number variations associate with resistance or susceptibility to gastrointestinal nematodes in Angus cattle. Funct. Integr. Genomics 12:81-92.

Hughes, J. F., and D. C. Page. 2015. The biology and evolution of mammalian Y chromosomes. Annu. Rev. Genet. 49:507-527.

Iannuzzi, L., W. A. King, and D. Di Berardino. 2009. Chromosome evolution in domestic bovids as revealed by chromosome banding and FISH-mapping techniques. Cytogenet. Genome Res. 126:4962.

Justel, A., D. Peña, and R. Zamar. 1997. A multivariate KolmogorovSmirnov test of goodness of fit. Stat. Probab. Lett. 35:251-259.

Kapustin, Y., A. Souvorov, T. Tatusova, and D. Lipman. 2008. Splign: Algorithms for computing spliced alignments with identification of paralogs. Biol. Direct 3:20.

Klopocki, E., and S. Mundlos. 2011. Copy-number variations, noncoding sequences, and human phenotypes. Annu. Rev. Genomics Hum. Genet. 12:53-72.

Laity, J. H., B. M. Lee, and P. E. Wright. 2001. Zinc finger proteins: new insights into structural and functional diversity. Curr. Opin. Struct. Biol. 11:39-46.

Letaief, R., E. Rebours, C. Grohs, C. Meersseman, S. Fritz, L. Trouilh, D. Esquerre, J. Barbieri, C. Klopp, R. Philippe, V. Blanquet, D. Boichard, D. Rocha, and M. Boussaha. 2017. Identification of copy number variation in French dairy and beef breeds using next-generation sequencing. Genet. Sel. Evol. 49:77.

Li, G., B. W. Davis, T. Raudsepp, A. J. Pearks Wilkerson, V. C. Mason, M. Ferguson-Smith, P. C. O'Brien, P. D. Waters, and W. J. Murphy. 2013. Comparative analysis of mammalian Y chromosomes illuminates ancestral structure and lineage-specific evolution. Genome Res. 23:1486-1495.

Mann, H. B., and D. R. Whitney. 1947. On a test of whether one of two random variables is stochastically larger than the other. Ann. Math. Stat. 18:50-60.

Noce, T., Y. Fujiwara, M. Sezaki, H. Fujimoto, and T. Higashinakagawa. 1992. Expression of a mouse zinc finger protein gene in both spermatocytes and oocytes during meiosis. Dev. Biol. 153:356-367.

Oluwole, O. A., K. Mahboubi, L. A. Favetta, T. Revay, T. Kroetsch and W. A. King. 2017. Highly dynamic temporal changes of TSPY gene copy number in aging bulls. PLoS One 12:e0178558.

Pieler, T., and E. Bellefroid. 1994. Perspectives on zinc finger protein function and evolution - An update. Mol. Biol. Rep. 20:1-8.

Sambrook, J., D. Russell, P. Maccallum, and D. Russell. 2001. Molecular Cloning: A Laboratory Manual, 3rd ed. Immunology 49:895909.

Schmittgen, T. D., and K. J. Livak. 2008. Analyzing real-time PCR data by the comparative C(T) method. Nat. Protoc. 3:1101-1108.

Shapiro, S. S., and M. B. Wilk. 1965. An analysis of variance test for normality (complete samples). Biometrika 52:591-611.

Shen, Y., Y. Yan, Y. Liu, S. Zhang, D. Yang, P. Zhang, L. Li, Y. Wang, Y. Ma, D. Tao, and Y. Yang. 2013. A significant effect of the TSPY1 copy number on spermatogenesis efficiency and the phenotypic expression of the gr/gr deletion. Hum. Mol. Genet. 22:1679-1695.

Shi, W., A. Massaia, S. Louzada, R. Banerjee, P. Hallast, Y. Chen, A. Bergstrom, Y. Gu, S. Leonard, M. A. Quail, Q. Ayub, F. Yang, C. Tyler-Smith, and Y. Xue. 2018. Copy number variation arising 
from gene conversion on the human Y chromosome. Hum. Genet. 137:73-83.

Skaletsky, H., T. Kuroda-Kawaguchi, P. J. Minx, H. S. Cordum, L. Hillier, L. G. Brown, S. Repping, T. Pyntikova, J. Ali, T. Bieri, A. Chinwalla, A. Delehaunty, K. Delehaunty, H. Du, G. Fewell, L. Fulton, R. Fulton, T. Graves, S.-F. Hou, P. Latrielle, S. Leonard, E. Mardis, R. Maupin, J. McPherson, T. Miner, W. Nash, C. Nguyen, P. Ozersky, K. Pepin, S. Rock, T. Rohlfing, K. Scott, B. Schultz, C. Strong, A. Tin-Wollam, S.-P. Yang, R. H. Waterston, R. K. Wilson, S. Rozen, and D. C. Page. 2003. The male-specific region of the human $\mathrm{Y}$ chromosome is a mosaic of discrete sequence classes. Nature 423:825-837.

Soh, Y. Q., J. Alfoldi, T. Pyntikova, L. G. Brown, T. Graves, P. J. Minx, R. S. Fulton, C. Kremitzki, N. Koutseva, J. L. Mueller, S. Rozen, J. F. Hughes, E. Owens, J. E. Womack, W. J. Murphy, Q. Cao, P. de Jong, W. C. Warren, R. K. Wilson, H. Skaletsky, and D. C. Page. 2014. Sequencing the mouse Y chromosome reveals convergent gene acquisition and amplification on both sex chromosomes. Cell 159:800-813.

Song, S., N. Yao, M. Yang, X. Liu, K. Dong, Q. Zhao, Y. Pu, X. He, W. Guan, N. Yang, Y. Ma, and L. Jiang. 2016. Exome sequencing reveals genetic differentiation due to high-altitude adaptation in the Tibetan cashmere goat (Capra hircus). BMC Genomics 17:122.

Tamura, K., D. Peterson, N. Peterson, G. Stecher, M. Nei, and S. Kumar. 2011. MEGA5: Molecular evolutionary genetics analysis using maximum likelihood, evolutionary distance, and maximum parsimony methods. Mol. Biol. Evol. 28:2731-2739.

Trombetta, B., and F. Cruciani. 2017. Y chromosome palindromes and gene conversion. Hum. Genet. 136:605-619.

Yan, Y., X. Yang, Y. Liu, Y. Shen, W. Tu, Q. Dong, D. Yang, Y. Ma, and Y. Yang. 2017. Copy number variation of functional $R B M Y 1$ is associated with sperm motility: An azoospermia factor-linked candidate for asthenozoospermia. Hum. Reprod. 32:1521-1531.
Yang, Y., T.-C. Chang, H. Yasue, A. K. Bharti, E. F. Retzel, and W.-S. Liu. 2011. ZNF280BY and ZNF280AY: autosome derived Y-chromosome gene families in Bovidae. BMC Genomics 12:13.

Yue, X. P., T. C. Chang, J. M. DeJarnette, C. E. Marshall, C. Z. Lei, and W. S. Liu. 2013. Copy number variation of PRAMEY across breeds and its association with male fertility in Holstein sires. J. Dairy Sci. 96:8024-8034.

Yue, X. P., C. Dechow, T. C. Chang, J. M. Dejarnette, C. E. Marsahall, C. Z. Lei, and W. S. Liu. 2014. Copy number variations of the extensively amplified Y-linked genes, HSFY and ZNF280BY, in cattle and their association with male reproductive traits in Holstein bulls. BMC Genomics 15:113.

Zhang, F., W. Gu, M. E. Hurles, and J. R. Lupski. 2009. Copy number variation in human health, disease, and evolution. Annu. Rev. Genomics Hum. Genet. 10:451-481.

Zhang, G. W., J. Q. Guan, Z. G. Luo, W. X. Zhang, L. Wang, X. L. Luo, and F. Y. Zuo. 2016. A tremendous expansion of TSPY copy number in crossbred bulls (Bos taurus $\times$ Bos grunniens). J. Anim. Sci. 94:1398-1407.

Zhang, L., S. Jia, M. Plath, Y. Huang, C. Li, C. Lei, X. Zhao, and H. Chen. 2015. Impact of parental Bos taurus and Bos indicus origins on copy number variation in traditional Chinese cattle breeds. Genome Biol. Evol. 7:2352-2361.

Zhang, S., W. Qiu, H. Wu, G. Zhang, M. Huang, C. Xiao, J. Yang, C. Kamp, X. Huang, K. Huellen, Y. Yue, A. Pan, R. Lebo, A. Milunsky, and P. Vogt. 2001. The shorter zinc finger protein ZNF230 gene message is transcribed in fertile male testes and may be related to human spermatogenesis. Biochem. J. 359:721-727.

Zhou, J., B. Lemos, E. B. Dopman, and D. L. Hartl. 2011. Copy-number variation: The balance between gene dosage and expression in Drosophila melanogaster. Genome Biol. Evol. 3:1014-1024. 